\title{
Cecil Balmond
}

The Number 9: The Search for the Sigma Code

Munich: Prestel, 1999

Reviewed by Jay Kappraff

For ages, the properties of numbers have fascinated both professionals and laypersons. Number theory has long been recognized as perhaps the only area of mathematics in which amateurs have made significant contributions. The interest in number lies far beyond mere utilitarian applications. There is mystery to the countless patterns which emerge from their study. The distributions of primes have both a randomness and a kind of hidden order to them much as the stars in the sky. I recall a friend of my father's rhapsodizing about the discovery that he made as a child that the repeating digits of $1 / 7=.142857 \ldots$ when multiplied by $2,3,4,5$ and 6 produce numbers that are cyclic in their digits, i.e., $2 / 7=$ $.285714 \ldots, 3 / 7=.428571 \ldots$, etc. Cecil Balmond's paean to the number 9 entitled, Number 9: The Search for the Sigma Code is a masterpiece in presenting the wonder of number and pattern with loving simplicity.

Balmond's story unfolds as a young devotee of number, Enjil, attempts to attract the attention of his elders in a pursuit of the title "Master". In this quest, someone akin to a "fairy godmother" materializes from a moonbeam and provokes Enjil with a conundrum: "What is the fixed point of the wind?" This leads Enjil to an odyssey of discovery in which the number 9 plays the central role. The lady of the moonbeam importunes Enjil to "let your mind dwell on numbers! Take the most simple ones. Don't be afraid of the Elders; they are not bad men, but men with too much oldness stuffed into their brains. Take them back to their childhood, let them hop, skip, and jump through your constructions."

In fact the foundations of this book are familiar to professional mathematicians (the Elders) as the process of "casting out nines", where a number modulo 9 is determined by adding its digits; e.g., $34 \bmod 9=7$ while $345 \bmod 9=12 \bmod 9=3$. Professional mathematicians might be inclined to drop the subject here. However to do so would be to miss the many beautiful patterns and discoveries about number that are to be found by following the path of the number 9 wherever it leads. The key lies in a circular pattern based on multiplying the series 123456789 
successively by 1,2,3,4,5,6,7,8,9 modulo 9. Herein lies the "Sigma Code", sigma referring to the process of summing the digits of a number in order to write it as mod 9.

Mathematicians tend to think of number and geometry as being two distinct branches from the tree of mathematics. Even the Academy of Plato formulated the quadrivium in which number and geometry were considered to be separate endeavors. Balmond does a great service by showing that by considering these two branches as one illuminates the study of each. In the sigma code, numbers are viewed either statically on a wheel with nine orbits on which the axis of symmetry and the outer orbit are all 9s, or dynamically as outward and inward spirals in which the number 9 is "the fixed point of the wind".

This small volume contains more than a quaint tale, but presents some real discoveries. For example, the digits of the sequence 142857 that appear in the decimal expansion of $1 / 7$ sigma sum to nine, and Balmond shows that other cyclic numbers also have the imprint of 9. Also this same sequence (but not in that order) appears cyclically in the sigma sums of the numbers in the geometric sequence: $1,2,4,8,16,32,64, \ldots$ The numbers of the sequence 142857 also represent the sigma sums of all of the prime numbers with the exception of 3 . However, the missing numbers in this sequence 3,6, and 9 also make their appearance in a mysterious six cycle of powers of each prime showing that primes are not random but subtly ordered. Each page brings new revelations involving Pascal's triangle, magic squares, the golden mean and other wonders relating number to geometry.

That our culture has also recognized the mysteries of the number 9 is suggested to us by Balmond who lists such proverbs as: "A stitch in time saves nine", "the whole nine yards", "to be on cloud nine", not to mention the 99 names of Allah. This book makes an entertaining read. It is an excellent way to interest young minds as to the wonders of number and invite non-mathematicians into the joy of making their own discoveries.

First published in the NNJ online January 2000

\section{The Reviewer}

Jay Kappraff holds a Ph.D. from the Courant Institute of Mathematical Science at New York University. He is an associate professor of mathematics at the New Jersey Institute of Technology where he has developed a course in the mathematics of design for architects and computer scientists. Prior to that, he taught at the Cooper Union College in New York City and held the position of aerospace engineer at NASA. He has published numerous articles on such diverse subjects as fractals, phyllotaxy, design science, plasma physics, passive solar heating and aerospace engineering. He has also lectured widely on the relationships between art and science. He is the author of Connections: The Geometric Bridge Between Art and Science. His new book, Mathematics Beyond Measure: A Random Walk Through Nature, Myth and Number, is to be published soon by World Scientific Press. 